\title{
A Methodology for Filtering and Inversion of Gravity Data: An Example of Application to the Determination of the Moho Undulation in Morocco
}

\author{
Victor Corchete $^{1}$, Mimoun Chourak ${ }^{2,3}$, Driss Khattach ${ }^{4}$ \\ ${ }^{1}$ Higher Polytechnic School, University of Almeria, Almeria, Spain \\ ${ }^{2}$ Faculté Polidisciplinaire d'Errachidia, University of Moulay Ismaill, B.P. Boutalamine, Morocco \\ ${ }^{3}$ NASG (North Africa Seismological Group), Gelderland, The Netherlands \\ ${ }^{4}$ Faculté des Sciences, University of Mohamed I, Oujda, Morocco \\ Email: corchete@ual.es
}

Received November 20, 2009; revised December 19, 2009; accepted December 22, 2009

\begin{abstract}
In this paper, we perform a comprehensive explanation of the geophysical inversion of gravity data, as well as, how it can be used to determine the Moho undulation and the crustal structure. This inversion problem and the necessary assumptions to solve it will be described in this paper joint to the methodology used to invert gravity data (gravity anomalies). In addition, the application of this method to the determination of the Moho undulation will be performed computing the Moho undulation in the Moroccan area, as an example. Before the inversion, it is necessary the removing of the gravity effects for shallow and very deep structures, to obtain the deep gravity anomaly field that is associated to the deep structure and Moho undulation. These effects will be removed by a filtering process of the gravity anomaly field and by subtraction of the gravity anomalies corresponding to the very deep structure. The results presented in this paper will show that the inversion of gravity data is a powerful tool, to research the structure of the crust and the upper mantle. By means of this inversion process, the principal structural features beneath of Morocco area will be revealed.
\end{abstract}

Keywords: FFT, Inversion, Bouguer Anomaly, Moho, Morocco

\section{Introduction}

As all of us know, geophysical inversion of the gravity data (gravity anomalies) can be used to determine the Moho undulation, as well as, the crustal structure. This inverse problem is an ill-posed problem because it has non-unique solution. Nevertheless, this non-uniqueness can be overcome if we taken into account restrictive (and reasonable) assumptions on the admissible density distribution. These assumptions can be provided by the geology of the study area, as well as, all geophysical and deep sounding data available for this area. For a comprehensive explanation of this inversion problem and the necessary assumptions to solve it, in this paper the methodology to invert gravity anomaly data will be described and an example of application of this method will be presented. In this example, the determination of the Moho undulation in the Moroccan area will be performed. Before the inversion, the gravity effects of shal- low structure must be removed, to obtain the deep gravity anomaly field, which is associated to the deep structure and Moho undulation. These effects can be removed performing a filtering process of the gravity anomaly field, as it will be described below. The gravity effects of structures deeper than the lithosphere also must be eliminated. These effects can be eliminated by subtraction of the gravity anomalies corresponding to this very deep structure. These gravity anomalies can be obtained from a worldwide gravity field model as the EGM2008 model.

\section{Data Set}

Land and marine gravity data bank. The National Geophysical Data Centre (NGDC), the Bureau Gravimetrique International (BGI) and the United Status Geological Survey (USGS); have provided the gravity data used in this study: simple Bouguer anomalies (Bouguer anomalies without terrain correction). NGDC contributes with a 
data set consisting of 40370 points (only marine data), the BGI data set in the study area has 46653 points (6096 in land and 40557 at the sea) and USGS supplies 70628 points all in land. These gravity data are distributed in the study area (27 to 36 degrees on latitude and -14 to -1 degrees on longitude) as it is shown in the Figure 1.The accuracy of these data ranges from 0.1 to $0.2 \mathrm{mgal}$. The compiling gravity data have been checked to remove repeated points letting 110998 points, with a Bouguer anomaly range from -164 to $350 \mathrm{mgal}$. All gravity data have been converted to the same reference system (GRS 80 reference system) and the atmospheric correction has been taken into account [1].

Geopotential model. The EGM2008 geopotential model represents a major advance in modelling the Earth's gravity and geoid [2]. Therefore, the EGM2008 model is the model more suitable for the computing of the longwavelength contribution of the gravity anomaly field, to obtain high precision in the determination of this contribution for the study area.

Digital terrain model (DTM). The calculation of complete Bouguer anomalies from simple Bouguer anomalies involves the computation of the terrain correction, because complete Bouguer anomaly is simple Bouguer anomaly plus terrain correction, but terrain correction is achieved using a DTM (digital terrain model). For this reason, we need a DTM for the study area. This DTM will be computed using all the elevation data available for the study area: trackline bathymetry (from the NGDC and BGI), ETOPO1 and 3" gridded topography (from SRTM project); as it will be described in the next section. ETOPO1 is a 1-minute gridded global relief of combined bathymetry and topography, available from NGDC global database. SRTM topography is a worldwide elevation model with a 3" $\times 3$ " mesh size obtained from the Shuttle Radar Topography Mission (SRTM). This topography will be supplied by the USGS database.

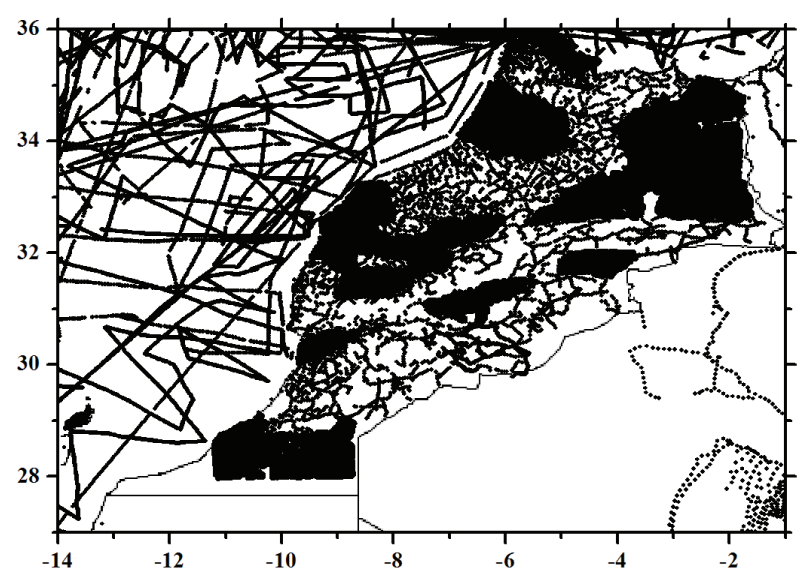

Figure 1. Distribution of gravity data over the study area.

\section{Data Preparation and Pre-Processing}

Bouguer anomaly data gridding. As it is well known, gravity anomalies on a regular grid is required for filtering by means of the FFT technique. Since the gravity data set consisting of point data anomalies distributed randomly, we need to interpolate these data to obtain a regular data grid. A precision interpolation procedure must provide a regular grid data with a high level of guarantee and reliability. In this interpolation process, it is necessary to reject some point data in which the difference between the observed and computed gravity anomaly value is greater than some reference value (50 mgal). We have used the OriginLab software package (C) 1991-2003 OriginLab Corporation) for the computation of a regular data grid, from the Bouguer gravity anomalies randomly distributed. This software package follows the kriging method to carry out the interpolation task [3]. The interpolation process is tested by means of the comparison, for each observed point, between the observed and predicted anomaly value. In a first step of this calculation process, it is necessary to reject the spike values always present in any Bouguer anomaly data set. Otherwise, it can arise abnormal and no sense grids during the interpolation process. For that reason, in the first step, the observed point values, in which the difference between observed and predicted value is greater than 50 mgal, have been rejected. By means of this validation procedure we have rejected 230 points from the original data set, letting 110768 points for the second step of the interpolation process. In the second step, we finished the interpolation process obtaining the gridded data, with a mean and standard deviation of the differences between the observed and predicted values of $0.2 \mathrm{mgal}$ and 4.5 mgal, respectively. Thus, the gridded data are distributed for the study area from 27 to 36 degrees of latitude and -14 to -1 degrees of longitude (east positive), in a $360 \times$ 520 regular grid with a mesh size of $1.5^{\prime} \times 1.5^{\prime}$ and 187200 points.

Computation of a DTM for the study region. As it was above mentioned, the terrain correction is necessary to calculate complete Bouguer anomalies and this terrain correction is obtained from a DTM. For our study area, we have a suitable gridded topography with a mesh size of 3" × 3" from SRTM project, but we have not a bathymetry with the same resolution. Then, we need compute this gridded bathymetry from the bathymetry data available for this area: trackline bathymetry (from NGDC and BGI) and ETOPO1. We have interpolated all these bathymetric data by kriging method, using the same software package above mentioned for interpolation of the gravity data. Thus, we combined gridded topography from SRTM project with gridded bathymetry obtained by interpolation, to make a DTM for our study 
region with a $3^{\prime \prime} \times 3^{\prime \prime}$ spacing $(90 \mathrm{~m} \times 90 \mathrm{~m}$, approximately). Figure 2 shows the flow chart of the process followed to make this DTM for the study region. Figure 3 shows the DTM resulting of the computation process followed.

Terrain correction and complete Bouguer anomaly computation. The necessary terrain correction was computed by means of a classical integration formula, as it is described by Torge [4]. The result obtained is shown in Figure 4. We have considered a density for the topographic masses of $2.67 \mathrm{~g} / \mathrm{cm}^{3}$ and $1.03 \mathrm{~g} / \mathrm{cm}^{3}$ for the seawater. The integration has been extended to 0.75 degrees (83 km, approximately) out of the study area to avoid the border effects. Finally, when we add the terrain correction (shown in Figure 4) to the simple Bouguer anomalies (gridded as it was above described), we obtain the complete Bouguer gravity anomalies to be used in this study. These gridded Bouguer anomalies are plotted in Figure 5.

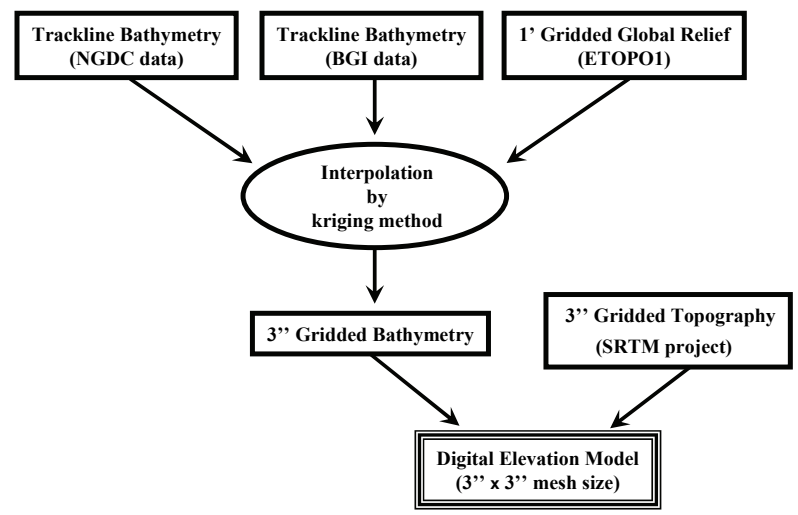

Figure 2. Steps followed to make the digital terrain model (DTM). The circles are used to denote the application of interpolation and the rectangles are used to denote the results obtained.

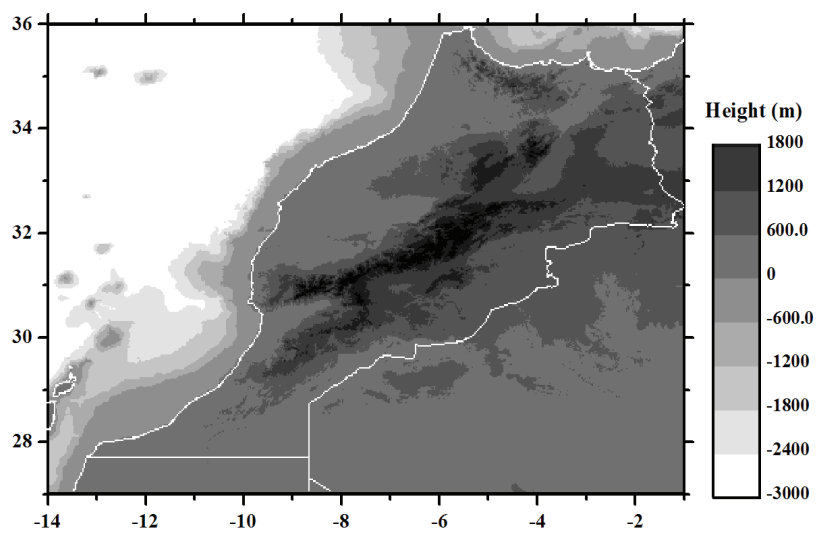

Figure 3. DTM (with a mesh size of 3 " $\times 3$ "') obtained as result of the computation process shown in Figure 2.

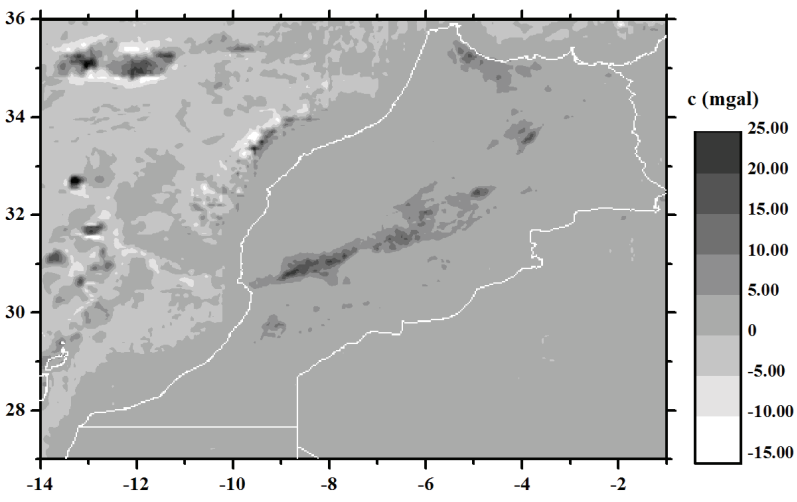

Figure 4. Terrain correction $c$ with a mesh size of $1.5, \times 1.5$ computed by integration from the DTM shown in Figure 3.

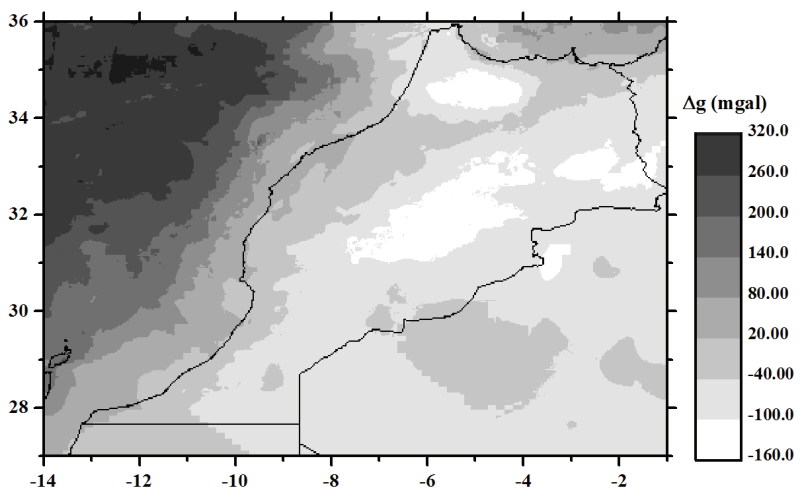

Figure 5. Complete Bouguer anomalies gridded with a mesh size of 1.5 ' $\times 1.5$, obtained adding the terrain correction to the simple Bouguer anomalies (gridded to the same mesh size).

\section{Short Wavelength and Long Wavelength Corrections}

Wavelength filtering. Before the inversion, we need to remove shallow and local effects in the anomaly gravity field, to obtain the deep gravity anomaly field, which is associated to Moho undulation. These effects are associated to the density irregularities distributed in the shallow structure of our study region. These effects can be eliminated after a filtering process of the gravity anomaly field, removing short wavelengths and remaining the long wave components of this field, which are associated to the deep structure that we want to analyse. This filtering can be done transforming the gridded Bouguer anomalies (Figure 5) in a 2D wave-number domain using 2D FFT, removing short wavelengths components by windowing with a suitable spectral window and, later, doing an inverse 2D FFT to reconstitute the Bouguer anomalies filtered (smoothed). A suitable window must produce minima distortion in both domains spatial and spectral. Using no particular window to remove short 
wavelengths, is equivalent to apply the rectangular window, which leads the spectral domain function undistorted but produces severe distortion in the spatial domain, associated to the sharp rise and fall of this window. Therefore, an engagement must be considered because a window that leaves both domains undistorted is impossible to find. We have applied a cosine-tapered rectangular window to filter short wavelengths components of the spectra [5]. This window is the most used because is flat over the most of spectral components, as a rectangular window, but tapers off avoiding the undesirable border effect associated to a rectangular window. Another problem with this filtering process is an undesirable effect called circular convolution (or cyclic convolution). This effect is an end-effect that occurs when sampled functions do not contain a sufficient number of zero values [6]. To avoid this effect, it is necessary the addition of 2D sampled function as many zeros as half of the width and height of the data area in all directions [7]. Figure 6 shows the complete Bouguer anomalies shown in Figure 5, after removing of the spectral components of wavelength less than 40', following the process detailed above. We can see a smoothed Bouguer anomaly map with the same resolution, of course, that the original Bouguer anomaly map $\left(1.5^{\prime} \times 1.5^{\prime}\right.$ mesh size $)$.

Long wavelength correction. The gravity effects of structures deeper than lithosphere must be also removed of the Bouguer anomalies before the inversion, to obtain the gravity anomalies associated to the lithosphere structure, which are the gravity data that we want to invert for the determination of the Moho undulation. These gravity effects are associated to the long-wavelength components of the worldwide gravity field models (as EGM 2008), with a degree less than 30 of the harmonic expansion [4]. These long-wavelength effects are assumed as originated from density anomalies in the derivative. The

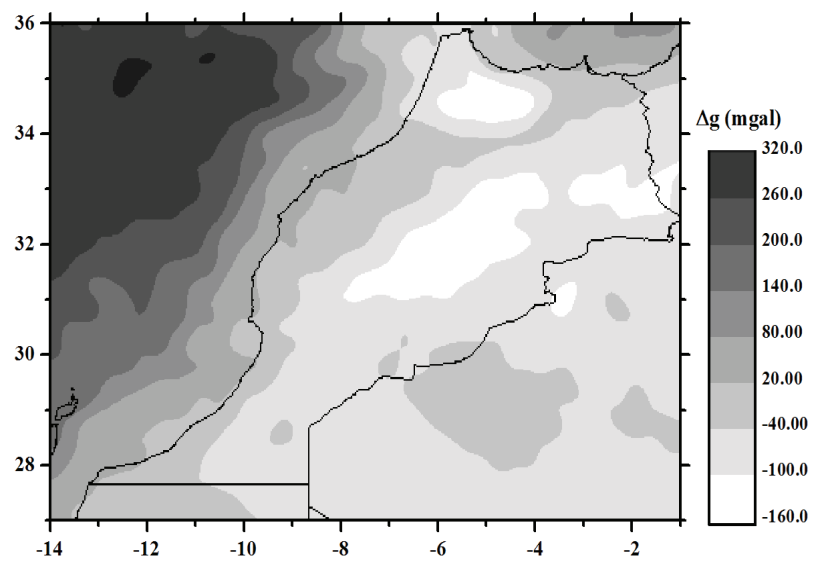

Figure 6. Bouguer anomaly map after filtered with a spectral window that removes wavelengths less than 40' (gridded to the same mesh size as Figure 5).
Earth structure below the lithosphere, and must be removed if we want to analyse only the lithosphere features. Figure 7 shows the gravity anomalies computed from EGM2008 model by means of the spherical harmonic expansion considered to degree and order $30[4,8]$.

Finally, we obtain Bouguer gravity anomalies, short and long wavelength corrected, subtracting the model gravity anomalies (shown in Figure 7) from the filtered gravity anomalies (shown in Figure 6). Figure 8 shows these corrected Bouguer anomalies, which reflect now the deep features of the lithosphere under the study region.

\section{Vening Meinesz Theory}

As it is well known, any inversion process requires an initial model. Inversion of gravity anomalies requires also a starting model to calculate the attraction and its

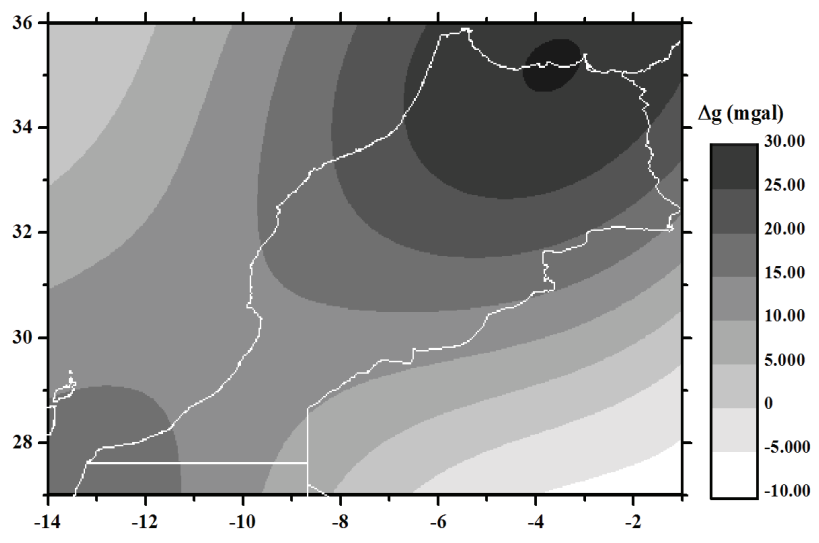

Figure 7. Gravity anomaly obtained from the Earth gravity model EGM2008, considering maximum degree and order equals to 30 (gridded to the same mesh size as Figure 6).

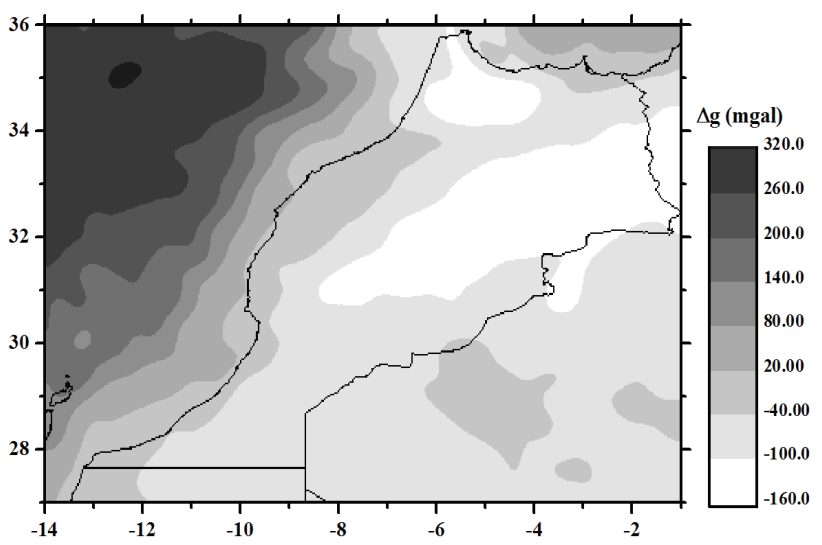

Figure 8. Bouguer anomaly map after the application of the filtering and long wavelength correction (gridded to the same mesh size as Figures 6 and 7). 
derivative of the attraction and the difference between theoretical gravity anomaly (obtained by forward modelling) and observed gravity anomaly (Bouguer anomalies shown in Figure 8), will be used to correct this initial model iteratively, obtaining a final Moho undulation that satisfy the gravity data (Figure 8). Thus, a starting model is necessary to start the inversion process. This model is constituted by a theoretical Moho undulation and a reasonable distribution of density. The density distribution is proposed from the geological and geophysical studies carried out in the study area. The theoretical Moho undulation can be computed applying the Vening Meinesz' theory of isostasy. As we know, the Vening Meinesz' theory is a modification of the Airy's theory of isostasy, introducing regional compensation instead of local compensation. Obviously, with this change the Vening Meinesz' theory is more realistic than the previous theory proposed by Airy, because Airy presuppose free vertical mobility of the crust masses, while Vening Meinesz considers the crust as an unbroken but yielding elastic layer. This yielding elastic layer can be bending by effect of the load of the topography. This bending can be calculated (following the Vening Meinesz' theory), from a detailed topography (as is given by a DTM), as a function of two constants $l$ (called by Vening Meinesz as degree of regionality) and $b$. Both constants are related by the Formula [9]

$$
b=\frac{1}{8\left(\rho_{1}-\rho_{2}\right) l^{2}}
$$

where $\rho_{0}$ is the crustal material density and $\rho_{1}$ is the mantle material density. The degree of regionality, denoted by $l$, has a length dimension and its values (in S.I. units) ranges from 10 to $60 \mathrm{~km}$. Small values of $l$ causes a Moho surface with minima more narrow and deeper, the opposite causes wide and shallow minima.

\section{Inversion of Gravity Data}

Forward method. In the inversion process, the direct problem of gravimetry (also called forward modelling) appears as it has been mentioned in the previous section. This direct problem consists of the calculation of the gravitational attraction, generated by some distribution of anomalous mass. This calculation procedure is usually called forward modelling and it is carried out by integration. This integration is computed over the volume of anomalous mass, considering the observation point on the surface of the study area (assumed flat). The gravity anomaly (the vertical component of the gravity attraction) is related to the density contrast by

$$
\Delta g=\int_{V} G \Delta \rho \frac{z}{r^{3}} d V
$$

where $\Delta \rho$ is the density contrast, $G$ is the Newton's gravitational constant, $z$ is the depth to the mass point and $r$ is the distance between the mass point and the observation point. This integral can be easily solved by numerical computation, if we assume that the volume of anomalous mass, denoted by $V$, can be divided in rectangular prisms $V_{j}$, with a constant density contrast $\Delta \rho_{j}$ in each prism. Thus, the above integral can be replaced by a sum of integrals over right rectangular prisms, that is to say

$$
\Delta g_{i}=\sum_{j=1}^{m} A_{i j} \Delta \rho_{j} \quad A_{\mathrm{ij}}=G \int_{V_{j}} \frac{z}{r^{3}} d V
$$

where now, we denote $\Delta g_{i}$ as the gravity anomaly at the observation point (on the surface of the study area assumed flat), with $i$ varying from 1 to $n$ observation points and $j$ varying from 1 to $m$ prisms. $A_{\mathrm{ij}}$ is a purely geometric term and it represents the attraction of a prism with density contrast unity. Such attraction has been calculated by Nagy [10]. Figure 9 shows a picture of the forward modelling. Thus, the forward modelling can be performed, if we have a starting model given by a reasonable distribution of density $\left(\Delta \rho_{j}\right.$ for $\left.\mathrm{j}=1,2, \ldots, \mathrm{n}\right)$ in a volume of anomalous mass $\left(V_{j}\right.$ for $\left.\mathrm{j}=1,2, \ldots, \mathrm{n}\right)$, obtaining after this calculation procedure a grid of gravity anomalies $\left(\Delta \mathrm{g}_{\mathrm{i}}\right.$ for $\left.\mathrm{i}=1,2, \ldots, \mathrm{n}\right)$ distributed over the study area.

Inverse method. The inverse problem of gravimetry has been defined as the computation of the density distribution from the observed gravity anomaly field. That is, formally, the inversion of the relationship (1) considering as data the gravity anomalies. If we assume that the density contrast is known without error for each prism (through the geology and/or other previous geophysical studies), the problem consists only in the determination of the volume and shape of this anomalous mass, i.e. the computation of the size for each prism $\left(V_{j}\right)$. Thus, if we take into account right rectangular prisms with a fixed base $S_{j}$ $=\left(x_{2}-x_{1}\right) \times\left(y_{2}-y_{1}\right)$ and variable height $h_{j}=z_{1}-z_{2}$, as it is showed in Figure 10, the unknowns $x_{j}$ correspond to the heights $h_{j}$ of these prisms, while the data $y_{i}$ of this problem are $\Delta \mathrm{g}_{\mathrm{i}}$, the ith observed Bouguer anomaly.

Obviously, no linear relation exists between data and unknowns, as they have been defined, but we can linearize (1) by a Taylor expansion about an initial model, which is denoted by $x_{j}^{0}$, in the way

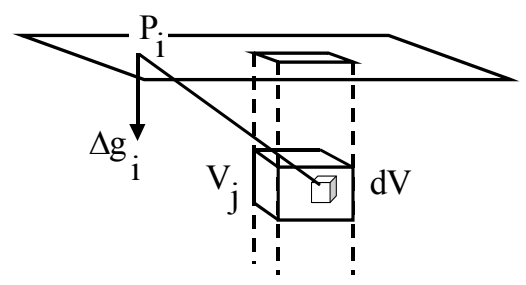

Figure 9. Attraction due to a right rectangular prism on an observation point over the surface of the study area. 


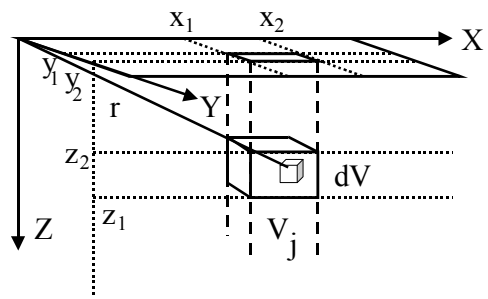

Figure 10. Attraction due to a right rectangular prism on a point located at the origin coordinates.

$$
y_{i}=\sum_{j=1}^{m} A_{i j}\left(x_{j}^{0}\right)+\left.\sum_{j=1}^{m} \frac{\partial A_{i j}}{\partial x_{j}}\right|_{x_{j}^{0}}\left(x_{j}-x_{j}^{0}\right)+\ldots
$$

where

$$
(\mathrm{i}=1,2, \ldots, \mathrm{n}) \quad y_{i}=\Delta g_{i} \quad \mathrm{x}_{\mathrm{j}}=h_{j} \quad A_{\mathrm{ij}}=\Delta \rho_{j} G \int_{V_{j}} \frac{z}{r^{3}} d V
$$

and neglecting the second and higher terms in (2) we can write

$$
y_{i}-\sum_{j=1}^{m} A_{i j}\left(x_{j}^{0}\right)=\sum_{j=1}^{m} \frac{\partial A_{i j}}{\partial x_{j}} \Delta x_{j} \Rightarrow \Delta \mathrm{y}_{\mathrm{i}}=\sum_{j=1}^{m} B_{i j} \Delta x_{j}
$$

where

$$
\begin{aligned}
& \Delta y_{i}=y_{i}-\bar{y}_{i} \quad \bar{y}_{i}=\sum_{j=1}^{m} A_{i j}\left(x_{j}^{0}\right) \\
& \Delta x_{j}=x_{j}-x_{j}^{0} \quad B_{i j}=\frac{\partial A_{i j}}{\partial x_{j}}
\end{aligned}
$$

A starting model will provide us: the density contrasts $\Delta \rho_{j}$ and heights $x_{j}^{0}$; through a known or supposed distribution of anomalous mass. By forward modelling, we can obtain the theoretical gravity anomaly $\bar{y}_{i}$, to compute after the vector $\Delta y_{i}$ with the differences between observed and theoretical data, as it is written in (3b). The matrix $B_{i j}$ is the partial derivative of the attraction generates by the $j$ th prism over the $i$ th observed point. This matrix can be calculated from the Nagy formula by partial derivation.

Thus, the problem consists in the inversion of (3a) to obtain the unknowns $\Delta x_{j}$ and later $x_{j}$ through the relationship

$$
x_{j}=x_{j}^{0}+\Delta x_{j} \quad(\mathrm{j}=1,2, \ldots, \mathrm{m})
$$

The main problem is then the inversion of (3a) or equivalently the inversion of the matrix relation

$$
\Delta \mathbf{y}=\mathbf{B} \Delta \mathbf{x}
$$

which is now a set of linear equations. The inverse matrix of $\mathbf{B}$ can be obtained by a well-known inverse method called generalized inversion [11,12]. The inverse matrix obtained after this inversion process is called damped least squares inverse and it is given by

$$
\mathbf{C}=\left(\mathbf{B}^{\mathbf{T}} \mathbf{B}+\alpha \mathbf{I}\right)^{-1} \mathbf{B}^{\mathbf{T}}
$$

where $\alpha$ is the damping parameter and $\mathbf{I}$ is the identity matrix. The parameter $\alpha$ is introduced because it stabilizes the solution of the inverse problem. The addition of this factor in the main diagonal of matrix $\mathbf{B}^{\mathbf{T}} \mathbf{B}$ increases the magnitude of its eigenvalues, avoiding the problems caused by small or zero eigenvalues that make the matrix $\mathbf{B}^{\mathrm{T}} \mathbf{B}$ singular or nearly singular. Substituting the relation called single value decomposition of $\mathbf{B}$

$$
\mathbf{B}=\mathbf{U}_{\mathrm{p}} \Lambda_{\mathrm{p}} \mathbf{V}_{\mathrm{p}}^{\mathrm{T}}
$$

in Formula (6), we can write

$$
\mathbf{C}=\mathbf{V}_{\mathrm{p}} \Lambda_{\mathrm{p}}\left(\Lambda_{\mathrm{p}}^{2}+\alpha \mathbf{I}\right)^{-1} \mathbf{U}_{\mathrm{p}}^{\mathbf{T}}
$$

where the matrixes $\mathbf{U}_{\mathrm{p}}, \Lambda_{\mathrm{p}}, \mathbf{V}_{\mathrm{p}}$; are described in detail by Aki and Richards [11]. The matrix $\mathbf{C}$ defined by Formula (7) is called the damped generalized inverse. The resolution matrix is obtained through the product of the matrixes $\mathbf{C}$ and $\mathbf{B}$, that is

$$
\mathbf{C B}=\mathbf{V}_{\mathrm{p}} \Lambda_{\mathrm{p}}^{2}\left(\Lambda_{\mathrm{p}}^{2}+\alpha \mathbf{I}\right)^{-1} \mathbf{V}_{\mathrm{p}}^{\mathbf{T}}
$$

and the error in the model parameters is given by the corresponding variance as (assuming that the data are statistically independent and the same behaviour for the model parameters)

$$
\sigma_{\mathrm{x}}^{2}=\sigma_{\mathrm{y}}^{2} \mathbf{V}_{\mathrm{p}} \Lambda_{\mathrm{p}}^{2}\left(\Lambda_{\mathrm{p}}^{2}+\alpha \mathbf{I}\right)^{-2} \mathbf{V}_{\mathrm{p}}^{\mathrm{T}}
$$

where $\sigma_{\mathrm{x}}{ }^{2}$ and $\sigma_{\mathrm{y}}{ }^{2}$ are the variances in model parameters and data, respectively. We can see in (8) and (9) the role developed by $\alpha$ in the damped inversion process. The introduction of this parameter degrades the resolution but stabilize the solution obtained, by means of the reduction of the variance. Thus, an engagement must be considered to choose the value of $\alpha$, because an increase of this value reduces error in the determination of the model parameters, at the expense of to sacrifice the resolution.

Numerical procedure of the inversion process. Figure 11 shows a flow chart of this calculation process. The starting data fixed in this numerical procedure are (for each prism): the density contrast $\left(\Delta \rho_{j}\right)$, the base of the prism $\left(S_{j}\right)$ and the initial height of the prism $\left(x_{j}^{0}\right)$. The observed Bouguer anomaly, $\Delta \mathbf{g}_{\mathbf{o b}}$ vector, is also fixed during all the calculation process. Thus, only the heights of the prisms are changed, through the $\Delta \mathbf{x}$ vector, during the calculation process performed to fit the observed data given by the $\Delta \mathbf{g}_{\mathbf{o b}}$ vector. The calculation process is concluded when the norm of the differences between theoretical and observed data is minimal. In Figure 11, forward modelling is carried out by Formula (1), the computing of partial derivatives of attraction is made from Nagy formula by partial derivation, and the computing of damped generalized inversion is carried out by Formula (7). The resolution and the error are computed by the 

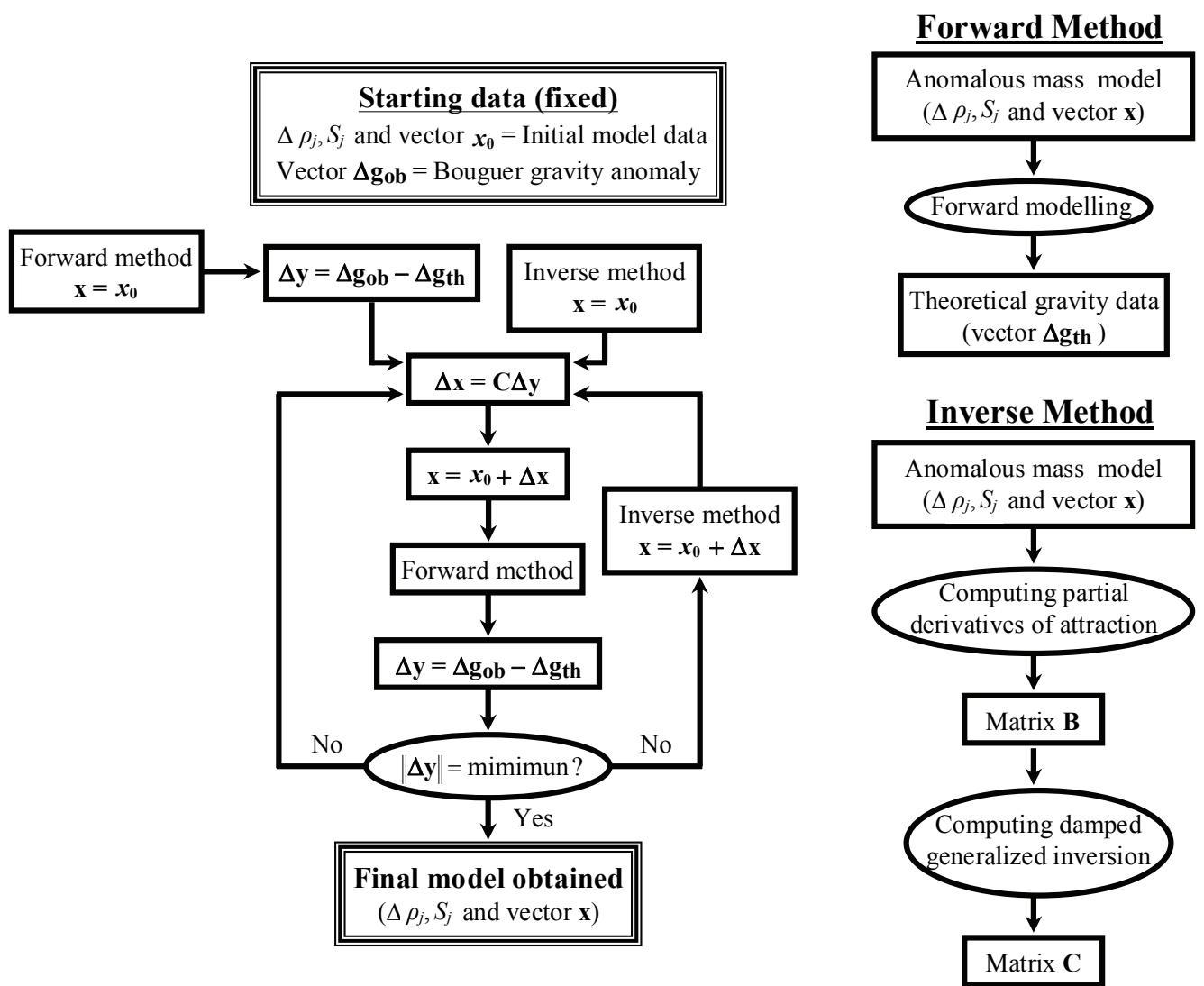

Figure 11. Numerical procedure followed in the inversion process. The circles are used to denote computation or control steps and the rectangles are used to denote methods or obtained results. The enhanced rectangles are used to show initial data and final result.

Formulas (8) and (9), respectively. The value of $\alpha$ is chosen as the value that produces less error for a resolution as good as possible.

\section{Moho Undulation Determination}

Sedimentary layer attraction. The wave number filtering performed in section 4 removes the short wavelength effects in the gravity data, but the wave-number spectrum of most shallow features, as the sedimentary cover of the study area, are broadband. Therefore, the spectrum of features at shallow depths overlaps spectrum of deep features (as Moho undulation). Consequently, the effects of these shallow features cannot be removed completely by filtering. This fact does necessary the computation of gravity effects due to these shallow features, by forward modelling. Later, these effects can be removed of the gravity anomaly field by subtraction, letting only the gravity effects due to the deep features. To do this, we need to collect all the available geophysical and geological information, to purpose a density distribution corresponding to these shallow features, as the sedimen- tary cover. Thus, the size of the study area is limited by the gravity data distribution and the geological and geophysical information, because the study area must be well covered by data to assure the reliability of the results obtained.

In Figure 12, we can see the study area divided in

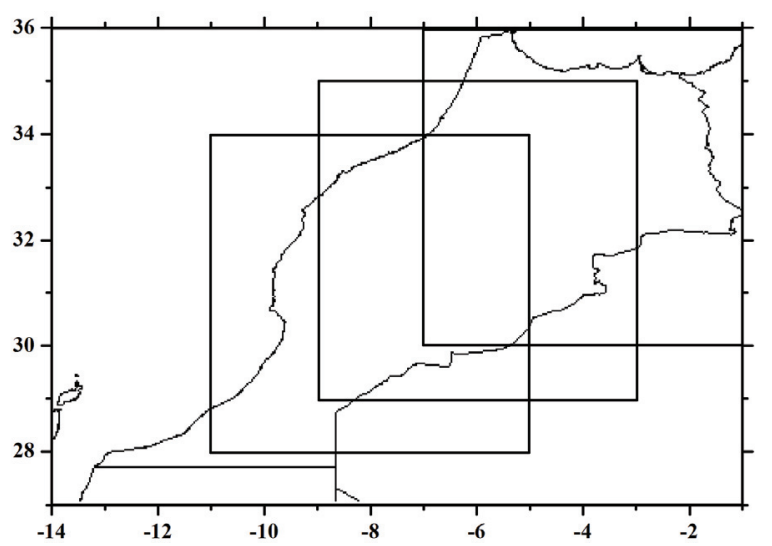

Figure 12. Study area divided in three overlapping zones with a size of $6 \times 6$ degrees. 
three overlapping zones that are fitted to the area covered by the gravity data, avoiding the zones with scarcity of data. Each zone with $6^{\circ} \times 6^{\circ}$ of size is divided in $30 \times 30$ prisms, as it is shown in Figure 13. We have then 900 prisms in each zone $\left(6^{\circ} \times 6^{\circ}\right)$, with the same base $0.2^{\circ} \times$ $0.2^{\circ}$ for each prism, but different height given by the thickness of the sedimentary layer, as it can be see in Figure 14. The corresponding attraction, shown in Figure 15, is calculated by forward modelling (as it was described in the previous section) with a density contrast of $0.34 \mathrm{~g} / \mathrm{cm}^{3}$. The density contrast is assumed constant for all the zones and prisms. This density contrast is obtained as the difference between the media density of the sediments $\left(2.38 \mathrm{~g} / \mathrm{cm}^{3}\right)$ and the media density for the upper crust $\left(2.72 \mathrm{~g} / \mathrm{cm}^{3}\right)$. The density values used in this modelling are given, for this study area, by Mickus and Jallouli [13].

Moho undulation. After the computation of the gravity attraction of the sedimentary cover, we remove this attraction from the gravity anomaly field shown in Figure 8, obtaining then the observed gravity data (vector $\Delta \mathbf{g}_{\mathbf{o b}}$ of the flow chart shown in Figure 11) needed for the inverse process. Figure 16 shows this gravity anomaly field (vector $\Delta \mathbf{g}_{\mathbf{o b}}$ ). Nevertheless, to start the inversion process (as it has been described in the previous section) is also necessary to have some initial model, constituted by an initial Moho undulation and its corresponding density contrast.

The density contrast of $0.34 \mathrm{~g} / \mathrm{cm}^{3}$ is assumed constant for all study area for depths greater than $20 \mathrm{~km}$ (media depth of the Conrad discontinuity). This density contrast is obtained as the difference between the lower-crust media density $\left(2.96 \mathrm{~g} / \mathrm{cm}^{3}\right)$ and upper-mantle media density $\left(3.30 \mathrm{~g} / \mathrm{cm}^{3}\right)$. For depths less than $20 \mathrm{~km}$, we consider a density contrast of $0.58 \mathrm{~g} / \mathrm{cm}^{3}$, which is obtained as the difference between the upper-crust media density $\left(2.72 \mathrm{~g} / \mathrm{cm}^{3}\right)$ and upper-mantle media density $(3.30$ $\left.\mathrm{g} / \mathrm{cm}^{3}\right)$. These values of density are given by Mickus and

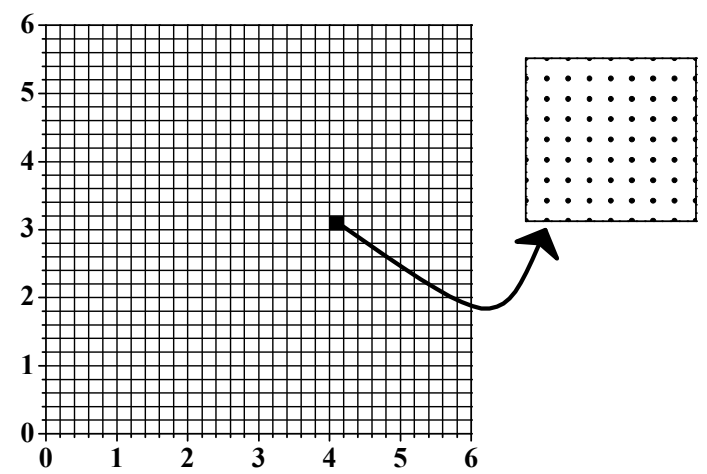

Figure 13. Each overlapping zone of $6^{\circ} \times 6^{\circ}$ (shown in Figure 12) is divided in $30 \times 30=900$ prisms with a base of $0.2^{\circ}$ $\times 0.2^{\circ}$. Each prism has $8 \times 8$ points data, if we cover the study area with a grid of $1.5, \times 1.5$ ' mesh size.

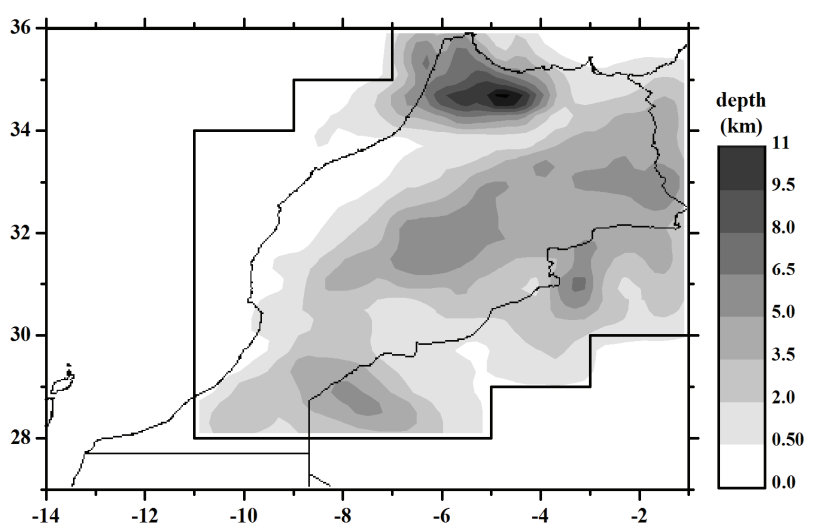

Figure 14. Thickness of the sedimentary cover (mesh size of $\left.0.2^{\circ} \times 0.2^{\circ}\right)$.

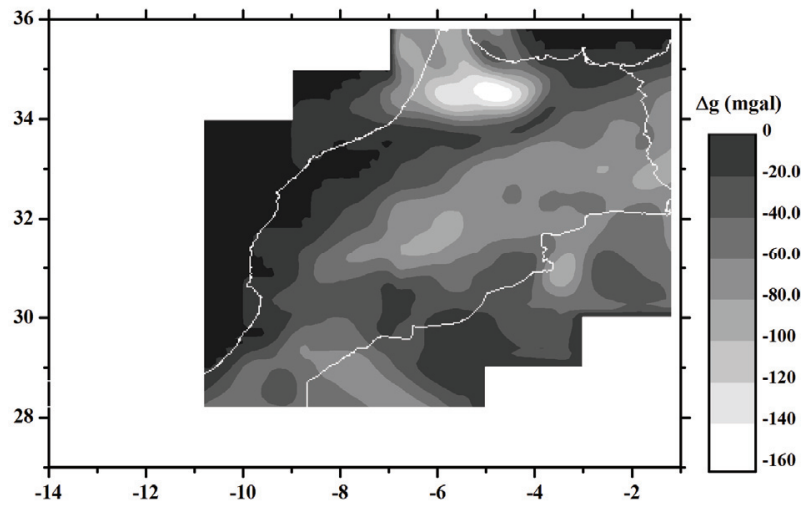

Figure 15. Gravity attraction of the sedimentary cover masses (mesh size of 1.5 ' $\times 1.5$ ').

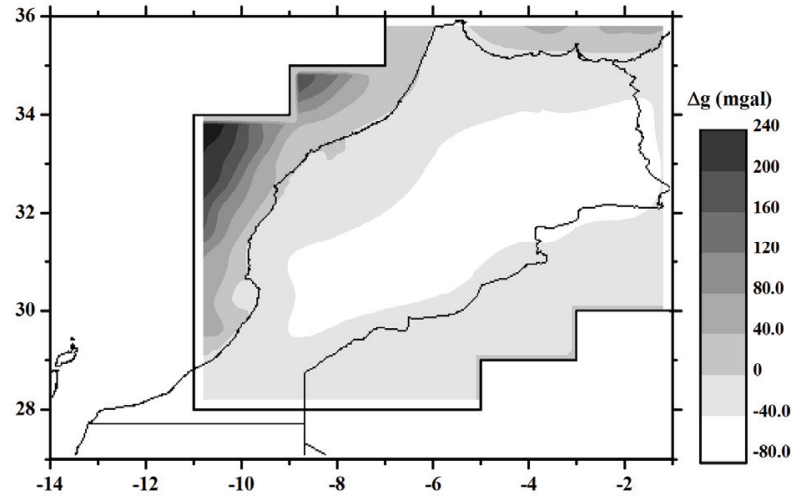

Figure 16. Bouguer anomaly field with the attraction of the sedimentary cover removed (mesh size of $1.5, \times 1.5$ ').

Jallouli [13], whose located the Conrad discontinuity at $20 \mathrm{~km}$ of media depth and the Moho discontinuity at 30 $\mathrm{km}$ of media depth.

Respect to the starting Moho undulation, we can find this initial surface applying the Vening Meinesz' theory 
of isostasy, as it was described in section 5, considering a suitable value for the degree of regionality. We take this value as $26 \mathrm{~km}$, because the Moho surface obtained for this value gives, through forward modelling, a gravity attraction field that is the most near to the observed gravity filed (as it can be seen by comparison between Figures 16 and 18). Figure 17 shows this starting Moho undulation obtained for the degree of regionality of 26 $\mathrm{km}$. Figure 18 shows the theoretical gravity anomaly obtained, by forward modelling, from this starting model. Once the starting data of the inversion process have been established, we can proceed to invert the gravity data as it was described in the previous section, obtaining the final Moho undulation shown in Figure 19. The errors in the Moho depth are computed by Formula (9). These errors raging between 0.02 and $0.7 \mathrm{~km}$ of standard deviation, but the major part of them are around $0.5 \mathrm{~km}$.

\section{Interpretation and Conclusions}

The results presented in this paper show that the inversion of gravity data is a powerful tool, to investigate the structure of the crust and upper mantle. By means of this technique are revealed the principal structural features beneath of Morocco. Such features are the existence of lateral and vertical heterogeneity in the study area, as it is concluded when we see Figure 20, in which a general picture of the lithospheric structure is shown from 0 to $50 \mathrm{~km}$. In this Figure, several Moho profiles performed in the study area show the different ways to make thin the crust.

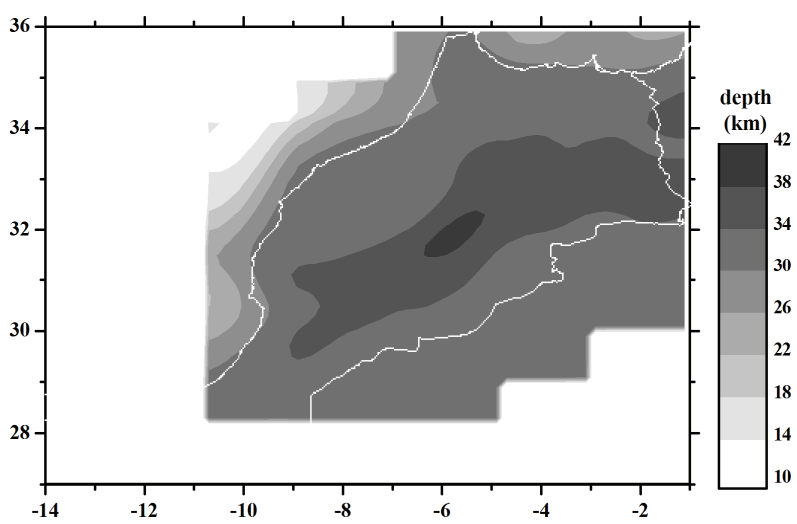

Figure 17. Moho depth predicted by Vening Meinesz' theory (mesh size of $\left.0.2^{\circ} \times 0.2^{\circ}\right)$.

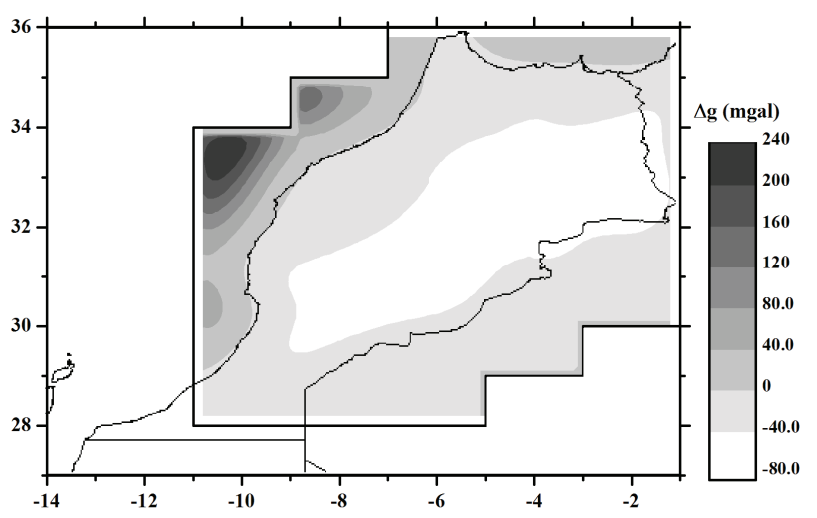

Figure 18. Theoretical Bouguer anomaly obtained by forward modelling from the starting model (mesh size of 1.5 ' $\times 1.5$ ').

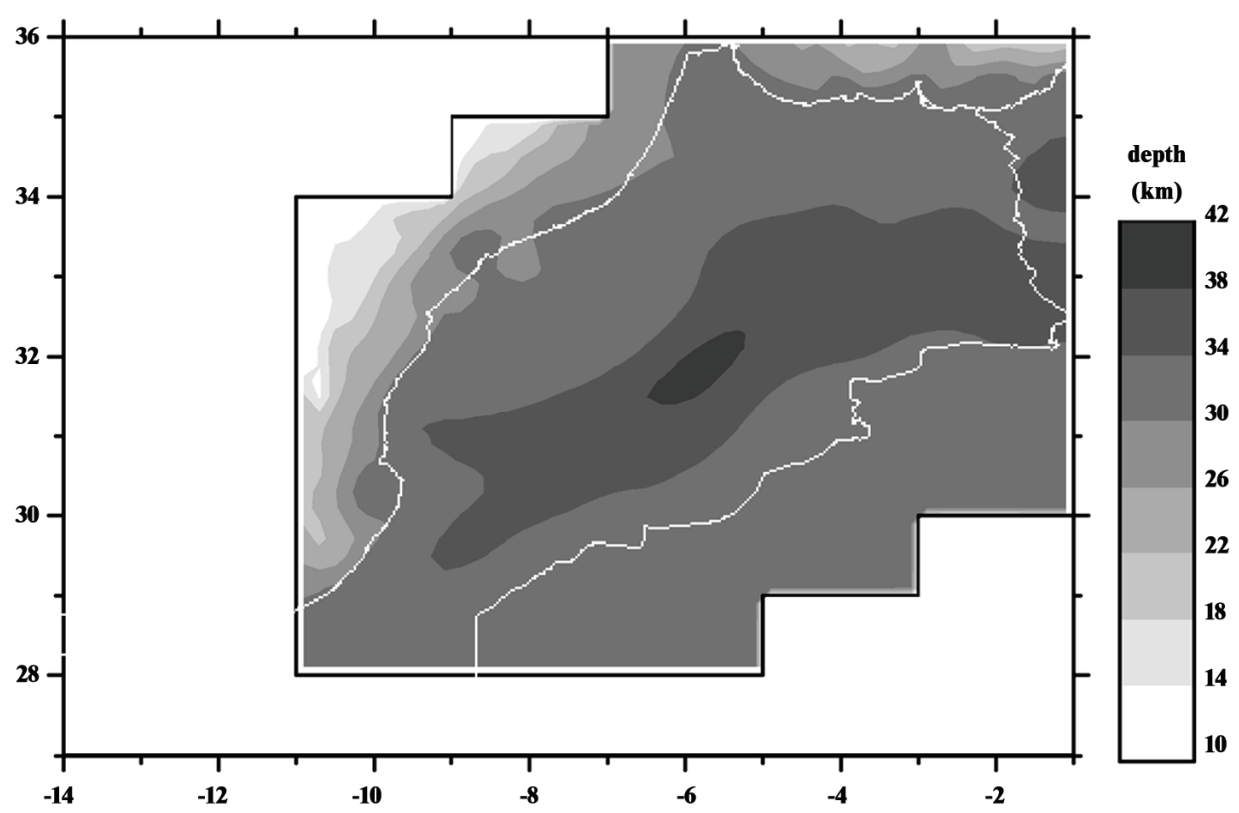

Figure 19. Moho depth distribution after the inversion process (mesh size of $\left.0.2^{\circ} \times 0.2^{\circ}\right)$. 

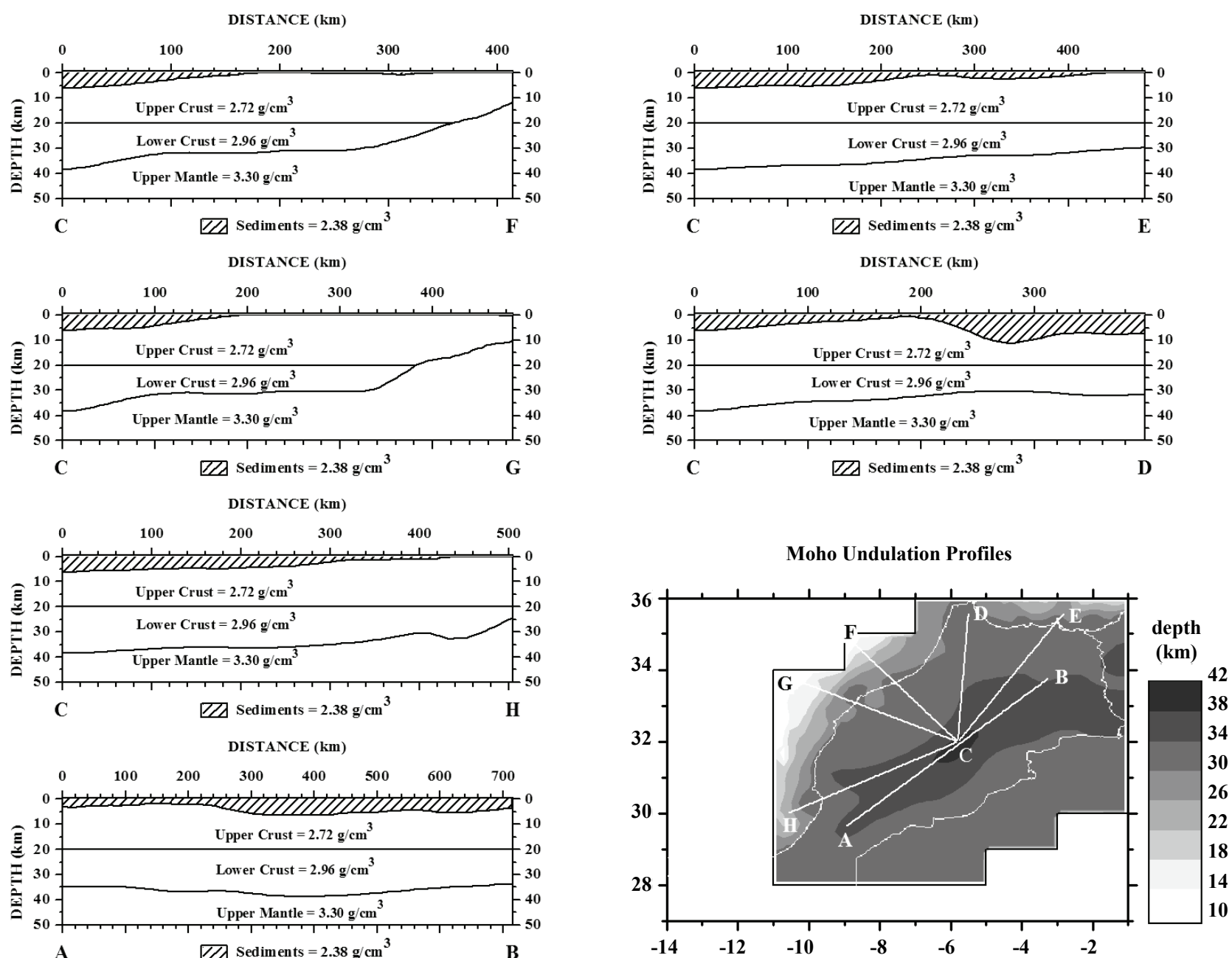

Figure 20. Crustal and upper mantle structure for several paths, showing the principal patterns of crustal thinning in Morocco.

\section{Acknowledgements}

The authors are grateful to the National Geophysical Data Center, the Bureau Gravimetrique International and the United States Geological Survey; whose have provided the gravity data used in this study. David Dater, Dan Metzger and Allen Hittelman (U.S. Department of Commerce, National Oceanic and Atmospheric Administration) have compiled the NGDC gravity data set. NGDC and USGS also have supplied the elevation data required to compute the necessary terrain correction.

\section{References}

[1] C. Wichiencharoen, "FORTRAN programs for computing geoid undulations from potential coefficients and gravity anomalies," Internal Report, Department of Geodetic Science and Surveying Report, Ohio State Univer- sity, Columbus, 1982.

[2] N. K. Pavlis, S. A. Holmes, S. C. Kenyon and J. K. Factor, "An Earth gravitational model to degree 2160: EGM 2008," Presented at the 2008 General Assembly of the European Geosciences Union, Vienna, Austria, pp. 13-18, April 2008.

[3] J. C. Davis, "Statistics and data analysis in Geology," John Wiley \& Sons Incorporation, 2nd edition, 1986.

[4] W. Torge, "Gravimetry," Walter de Gruyter, Berlin, New York, 1989.

[5] M. Bath, "Spectral analysis in Geophysics," Elsevier Scientific Publishing Company, 1974.

[6] O. E. Brigham, "The fast Fourier transform and its applications," Prentice Hall Signal Processing Series, 1988.

[7] R. Haagmans, E. de Min and M. van Gelderen, "Fast evaluation of convolution integrals on the sphere using 1D-FFT, and a comparison with existing methods for Stokes' integra," Manuscripta Geodaetica, Vol. 18, pp. 82-90, 1993. 
[8] W. A. Heiskanen and H. Moritz, "Physical geodesy," W. H. Freeman, San Francisco, 1967.

[9] H. Moritz, "The figure of the Earth. Theoretical geodesy and the Earth's interior," Wichmann, Berlin, 1990.

[10] P. Nagy, "The gravitational attraction of a right rectangular prism," Geophysics, Vol. 31, pp. 362-371, 1966.

[11] K. Aki and P. G. Richards, "Quantitative seismology. Theory and methods," W. H. Freeman and Company, San Francisco, 1980.
[12] V. Dimri, "Deconvolution and inverse theory. Application to geophysical problems. Methods in geochemistry and geophysics," Elsevier Science Publishers, Amsterdam, Vol. 29, 1992.

[13] K. Mickus and C. Jallouli, "Crustal structure beneath the tell and atlas mountains (algeria and tunisia) through the analysis of gravity data," Tectonophysics, Vol. 314, pp. 373-385, 1999. 